\title{
UNBOUNDED PERTURBATIONS OF FORCED HARMONIC OSCILLATIONS AT RESONANCE
}

\author{
TUNG-REN DING
}

\begin{abstract}
In 1969, A. C. Lazer and D. E. Leach proved an existence theorem for periodic solutions of Duffing's equations with bounded perturbations at resonance. In the present note, with the use of a topological technique, the author extended some results of Lazer and Leach to an $n$-dimensional Duffing system with unbounded perturbations at resonance.
\end{abstract}

1. In [1] A. C. Lazer proved an existence theorem for periodic solutions of a second-order equation of Duffing's type

$$
d^{2} x / d t^{2}+C(d x / d t)+g(x)=p(t),
$$

where $g(x)$ is such that $x g(x) \geqslant 0$ for $|x|$ sufficiently large and $g(x) / x \rightarrow 0$ as $|x| \rightarrow \infty$. In 1972 J. Mawhin [2] extended Lazer's result to an $n$-dimensional Liénard system

$$
\frac{d^{2} x}{d t^{2}}+\frac{d}{d t}[\operatorname{grad} F(x)]+g(x)=p(t),
$$

with conditions upon $g(x)$ analogous to those of Lazer (but in fact less severe).

In another paper [3], A. C. Lazer and D. E. Leach proved the existence of periodic solutions for Duffing's equation at resonance,

$$
d^{2} x / d t^{2}+m^{2} x+h(x)=p(t)
$$

with bounded perturbations $h(x)$ and some other conditions.

In the present note, with the use of a topological technique used in [5 and 6], we propose to prove the existence of periodic solutions of an $n$-dimensional Duffing system at resonance,

$$
d^{2} x_{s} / d t^{2}+m_{s}^{2} x_{s}+f_{s}(t, x)=p_{s}(t)
$$

$(s=1,2, \ldots, n)$ with unbounded perturbations $f_{s}(t, x)\left(x=\left(x_{1}, x_{2}, \ldots, x_{n}\right)\right)$ and some reasonable conditions, not much different from those in [2,3 and 4]. It will always be assumed in the sequel that $m_{s}>0$ are integers, $p_{s}(t) \in C(R, R) 2 \pi$-periodic in $t$, and $f_{s}(t, x) 2 \pi$-periodic in $t$ and continuously differentiable with respect to $x$.

Received by the editors October 29, 1981 and, in revised form, March 10, 1982.

1980 Mathematics Subject Classification. Primary 34C15, 34C25.

Key words and phrases. Duffing's equations, high dimension, resonance, unbounded perturbations, periodic solutions, existence theorem, topological technique. 
2. Before proceeding to the proof of our main results we require some lemmas:

Let $D_{s}$ be the closed unit disk centered at the origin $O_{s}$ of the Euclidean plane $R_{s}^{2}$, for $s=1,2, \ldots, n$. We then consider the topological product

$$
G=D_{1} \times D_{2} \times \cdots \times D_{n} \subset R^{2 n},
$$

and its boundary $\partial G=\Gamma_{1} \cup \Gamma_{2} \cup \cdots \cup \Gamma_{n}$, in which

$$
\begin{aligned}
\Gamma_{1}=\partial D_{1} \times D_{2} \times \cdots \times D_{n}, \\
\Gamma_{2}=D_{1} \times \partial D_{2} \times \cdots \times D_{n}, \ldots, \Gamma_{n}=D_{1} \times D_{2} \times \cdots \times \partial D_{n} .
\end{aligned}
$$

Denote by $\langle\cdot, \cdot\rangle$ the scalar product of vectors, and $|\cdot|$ the norm of a vector; let $z_{s}=\left(x_{s}, y_{s}\right)$ be a point or a vector in $R_{s}^{2}$, and then let $z=\left(z_{1}, z_{2}, \ldots, z_{n}\right)$ be a point or a vector in $R^{2 n}$.

Consider now a continuous mapping $T: G \rightarrow R^{2 n}$ defined by $\left(z_{1}, z_{2}, \ldots, z_{n}\right) \mapsto$ $\left(w_{1}, w_{2}, \ldots, w_{n}\right)$. Then the following lemma is an immediate consequence of a more general fixed-point theorem proved in [6].

LEMMA 1. If the above-mentioned mapping $T$ satisfies the boundary condition

$$
\left\langle w_{s}-z_{s}, z_{s}\right\rangle \neq\left|z_{s}\right| \cdot\left|w_{s}-z_{s}\right|, \text { for } z \in \Gamma_{s} \text { and } s=1,2, \ldots, n,
$$

then $T$ has at least one fixed point in $G$.

Next, by a similar method used in [5], we can derive an asymptotic formula of a definite integral containing a positive small parameter $\varepsilon$.

LeMma 2. Let $\varepsilon$ be a positive small parameter, $A$ and $\delta<\frac{1}{2}$ be two positive constants, and

$$
J(\varepsilon)=\int_{-\pi / 2+\sigma}^{\pi / 2-\sigma} \cos \theta \cdot \tan ^{-1}\left(\frac{1-\delta}{\varepsilon} \cos \theta\right) d \theta
$$

where $\sigma=A \pi \varepsilon<\pi / 2$. Then the asymptotic formula

$$
J(\varepsilon)=\pi-\pi \varepsilon /(1-\delta)+O\left(\varepsilon^{2}\right)
$$

holds if $\varepsilon$ is small enough.

COROLlaRY. The asymptotic formula

$$
\int_{\pi / 2+\sigma}^{3 \pi / 2-\sigma} \cos \theta \cdot \tan ^{-1}\left(\frac{1-\delta}{\varepsilon} \cos \theta\right) d \theta=\pi-\frac{\pi \varepsilon}{1-\delta}+O\left(\varepsilon^{2}\right)
$$

holds if $\varepsilon$ is small enough.

3. To establish the existence theorems for periodic solutions of (1.4), we shall give some reasonable assumptions about the perturbations. The first one is a release from the boundedness restrictions on perturbations (cf. [3, 2]).

$\left(\mathrm{H}_{1}\right)$ There exists a positive constant $A$ and a positive function $\beta(x)$ tending to zero as $|x| \rightarrow \infty$ such that the inequalities

$$
\left|f_{s}(t, x)\right| \leqslant|x| \cdot \beta(x) \quad(s=1,2, \ldots, n),
$$

hold for $t \in[0,2 \pi]$ and $|x| \geqslant A$. 
Let $\lambda>1$ be a large parameter, and $u_{s}=x_{s} / \lambda$ for $s=1,2, \ldots, n$. Then the differential system (1.4) becomes

$$
d^{2} u_{s} / d t^{2}+m_{s}^{2} u_{s}+\lambda^{-1} f_{s}\left(t, \lambda u_{s}\right)=\lambda^{-1} p_{s}(t)
$$

$(s=1,2, \ldots, n)$. Let us now consider an auxiliary system

$$
d^{2} u_{s} / d t^{2}+m_{s}^{2} u_{s}+F_{s}(t, u, \varepsilon)=\varepsilon p_{s}(t)
$$

where

$$
F_{s}(t, u, \varepsilon)= \begin{cases}\varepsilon f_{s}(t, u / \varepsilon), & 0<\varepsilon \leqslant 1 \\ 0, & \varepsilon=0\end{cases}
$$

for $s=1,2, \ldots, n$.

It follows from $\left(\mathrm{H}_{1}\right)$ that $F_{s}(t, u, \varepsilon)$ is continuous with respect to $(t, u, \varepsilon)$ and $F_{s}(t, u, 0)=0$. Note that $F_{s}(t, u, \varepsilon)$ is $2 \pi$-periodic in $t$ and differentiable with respect to $u$ for any fixed $\varepsilon(0 \leqslant \varepsilon \leqslant 1)$. Hence, we can still prove the continuity of solutions of (3.2) with respect to the parameter $\varepsilon$ and the initial values.

In particular, when $\varepsilon=1 / \lambda$, the differential system (3.2) coincides with (3.1). We can put (3.2) in its equivalent form

$$
\frac{d u_{s}}{d t}=m_{s} v_{s}, \quad \frac{d v_{s}}{d t}=-m_{s} u_{s}-\frac{1}{m_{s}} F_{s}(t, u, \varepsilon)+\frac{\varepsilon}{m_{s}} p_{s}(t)
$$

$(s=1,2, \ldots, n)$. We then consider the initial conditions

$$
\text { when } t=0, u=\xi \text { and } v=\eta \text {, }
$$

where $\xi=\left(\xi_{1}, \xi_{2}, \ldots, \xi_{n}\right)$ and $\eta=\left(\eta_{1}, \eta_{2}, \ldots, \eta_{n}\right)$ are two parameter $n$-vectors. It can be seen that the initial-value problem (3.3) and (3.4) has a unique solution,

$$
u=u(t, \xi, \eta, \varepsilon), \quad v=v(t, \xi, \eta, \varepsilon) \quad(-\infty<t<\infty),
$$

which is continuous in $(t, \xi, \eta, \varepsilon)$ for all $t \in R, \xi \in R^{n}, \eta \in R^{n}$ and $\varepsilon(0 \leqslant \varepsilon \leqslant 1)$. Therefore, for any fixed $\varepsilon(0 \leqslant \varepsilon \leqslant 1)$, by setting

$$
\bar{u}=u(2 \pi, \xi, \eta, \varepsilon), \quad \bar{v}=v(2 \pi, \xi, \eta, \varepsilon),
$$

we obtain a mapping $T_{\varepsilon}: G \rightarrow R^{2 n}$, that is, $(\xi, \eta) \mapsto(\bar{u}, \bar{v})$ defined by (3.6), in which $(\xi, \eta)=\left(\xi_{1}, \eta_{1}, \xi_{2}, \eta_{2}, \ldots, \xi_{n}, \eta_{n}\right)$ and $(\bar{u}, \bar{v})=\left(\bar{u}_{1}, \bar{v}_{1}, \bar{u}_{2}, \bar{v}_{2}, \ldots, \bar{u}_{n}, \bar{v}_{n}\right)$.

For any integer $k(1 \leqslant k \leqslant n)$, let $\left(\xi_{\jmath}, \eta\right) \in \Gamma_{k}$, and then we can set $\xi_{k}=\cos \theta_{k}^{\circ}$, $\eta_{k}=\sin \theta_{k}^{\circ}\left(0 \leqslant \theta_{k}^{\circ}<2 \pi\right)$ and

$$
u_{k}(t, \xi, \eta, \varepsilon)=\gamma_{k} \cos \theta_{k}, \quad v_{k}(t, \xi, \eta, \varepsilon)=\gamma_{k} \sin \theta_{k},
$$

where $\gamma_{k}=\gamma_{k}(t, \xi, \eta, \varepsilon) \geqslant 0$ and $\theta_{k}=\theta_{k}(t, \xi, \eta, \varepsilon)$ are continuous in $(t, \xi, \eta, \varepsilon)$. Obviously, we have

$$
\gamma_{k}(t, \xi, \eta, 0)=1, \quad \theta_{k}(t, \xi, \eta, 0)=-m_{k} t+\theta_{k}^{\circ} .
$$

From the continuity of $\gamma_{k}$ and $\theta_{k}$ with respect to $(t, \xi, \eta, \varepsilon)$, together with (3.7), we conclude that, for any constant $\delta\left(0<\delta<\frac{1}{2}\right)$, a positive number $\varepsilon_{1}\left(\varepsilon_{1} \leqslant 1\right)$ can be found such that when $(\xi, \eta) \in \Gamma_{k}$, the inequality

$$
1-\delta \leqslant \gamma_{k}(t, \xi, \eta, \varepsilon) \leqslant 1+\delta
$$


holds for $0 \leqslant t \leqslant 4 \pi$ and $0 \leqslant \varepsilon \leqslant \varepsilon_{1}$; and furthermore, we have

$$
-2\left(m_{k}+1\right) \pi<\theta_{k}(2 \pi, \xi, \eta, \varepsilon)-\theta_{k}^{\circ}<-2\left(m_{k}-1\right) \pi
$$

for $0 \leqslant \varepsilon \leqslant \varepsilon_{1}$, and it follows from (3.3) that

$$
\frac{d \theta_{k}}{d t}=-m_{k}-\frac{1}{m_{k} \gamma_{k}} F_{k}(t, u, \varepsilon) \cos \theta_{k}+\frac{\varepsilon}{m_{k} \gamma_{k}} p_{k}(t) \cos \theta_{k} .
$$

Lemma 3. If $\left(\mathrm{H}_{1}\right)$ holds, then there is a positive constant $\varepsilon_{2}$ such that for any $\varepsilon$ $\left(0 \leqslant \varepsilon \leqslant \varepsilon_{2}\right)$ and $(\xi, \eta) \in \Gamma_{k}$,

$$
d \theta_{k} / d t<0 \quad \text { for } t \in[0,4 \pi] \quad(k=1,2, \ldots, n) .
$$

(This lemma is an immediate consequence of (3.10) and (3.8) together with the continuity of $F(t, u, \varepsilon), F(t, u, 0)=0$.)

Geometrically, Lemma 3 means that for any $\varepsilon\left(0 \leqslant \varepsilon \leqslant \varepsilon_{2}\right)$ and $(\xi, \eta) \in \Gamma_{k}$, the projection $\left(u_{k}, v_{k}\right) \in R_{k}^{2}$ of the motion (3.5) moves clockwise around the origin $O_{k}$ when $0 \leqslant t \leqslant 4 \pi$.

Finally, we stress the inequality (3.9) by putting it in the following form:

Lemma 4. If $\left(\mathrm{H}_{1}\right)$ holds, then there is a positive constant $\varepsilon_{1}$ such that for any $\varepsilon$ $\left(0 \leqslant \varepsilon \leqslant \varepsilon_{1}\right)$ and $(\xi, \eta) \in \Gamma_{k}$, the angular increment of the projection $\left(u_{k}, v_{k}\right) \in R_{k}^{2}$ of (3.5),

$$
\Delta \theta_{k}=\theta_{k}(2 \pi, \xi, \eta, \varepsilon)-\theta_{k}^{\circ}=-2 m_{k} \pi,
$$

whenever $\Delta \theta_{k}$ is an integral multiple of $2 \pi$.

4. Now let us give the other basic assumptions.

$\left(\mathrm{H}_{2}\right)$ There exist positive constants $A$ and $B_{j}(j=1,2, \ldots, n)$ such that when $t \in[0,2 \pi]$ and $\left|x_{j}\right| \geqslant A$, the inequalities

$$
x_{j} f_{j}(t, x) \leqslant-B_{j} x_{j} \tan ^{-1} x_{j} \quad(j=1,2, \ldots, n)
$$

hold.

$\left(\mathrm{H}_{3}\right)$ The constants $B_{j}$ in $\left(\mathrm{H}_{2}\right)$ satisfy

$$
B_{j}>E_{j}^{\circ}+F_{j}^{\circ} \quad(j=1,2, \ldots, n),
$$

where

$$
E_{j}^{\circ}=\frac{1}{2 \pi}\left|\int_{0}^{2 \pi} p_{j}(t) \cos m_{j} t d t\right|, \quad F_{j}^{\circ}=\frac{1}{2 \pi}\left|\int_{0}^{2 \pi} p_{j}(t) \sin m_{j} t d t\right| .
$$

It is obvious that $\left(\mathrm{H}_{3}\right)$ implies the existence of a constant $\delta\left(0<\delta<\frac{1}{2}\right)$ such that

$$
\frac{1-\delta}{(1+\delta)^{2}} B_{j}>E_{j}^{\circ}+F_{j}^{\circ} \quad(j=1,2, \ldots, n) .
$$

In what follows we shall take $\delta$ such that (4.1) holds.

LEMmA 5. If $\left(\mathrm{H}_{1}\right),\left(\mathrm{H}_{2}\right)$ and $\left(\mathrm{H}_{3}\right)$ hold, then there exists a positive constant $\varepsilon_{3}$ such that for any $\varepsilon\left(0<\varepsilon \leqslant \varepsilon_{3}\right)$ and any $(\xi, \eta) \in \Gamma_{k}$, the increment

$$
\Delta \theta_{k}>-2 m_{k} \pi \text { for } k=1,2, \ldots, n \text {. }
$$


Proof. By Lemma 3, for any $\varepsilon\left(0 \leqslant \varepsilon \leqslant \varepsilon_{2}\right)$ and $(\xi, \eta) \in \Gamma_{\mathrm{k}}$,

$$
d \theta_{k} / d t=-\Phi_{k}<0 \text { for } 0 \leqslant t \leqslant 4 \pi
$$

where

$$
\Phi_{k} \equiv m_{k}+\frac{1}{m_{k} \gamma_{k}} F(t, u(t), \varepsilon) \cos \theta_{k}-\frac{\varepsilon}{m_{k} \gamma_{k}} p_{k}(t) \cos \theta_{k}
$$

It follows that from $\theta_{k}=\theta_{k}(t, \varepsilon)$ we can solve $t=t\left(\theta_{k}, \varepsilon\right)$ which is continuous in $\left(\theta_{k}, \varepsilon\right)$ and differentiable with respect to $\theta_{k}$ for any fixed $\varepsilon\left(0 \leqslant \varepsilon \leqslant \varepsilon_{2}\right)$. Then we can think of $\Phi_{k}$ as a continuous function of $\left(\theta_{k}, \varepsilon\right)$.

Given $\varepsilon\left(0 \leqslant \varepsilon \leqslant \varepsilon_{2}\right)$ and $(\xi, \eta) \in \Gamma_{k}$, let $\tau_{k}$ be the time such that the projection $\left(u_{k}, v_{k}\right) \in R_{k}^{2}$ of the motion (3.5) will complete $m_{k}$ rotations around the origin $O_{k}$ during the time interval $\left[0, \tau_{k}\right]$. Hence, by $(3.10)$, we get

$$
\tau_{k}=\int_{\theta_{k}^{\circ}-2 m_{k} \pi}^{\theta_{k}^{\circ}} \frac{d \theta_{k}}{\Phi_{k}}
$$

which implies that $\tau_{k}=\tau_{k}(\varepsilon)$ is continuous in $\varepsilon$. We note that $\tau_{k}(0)=2 \pi$. Set

$$
\tau_{k}=\sum_{j=0}^{m_{k}-1}\left(I_{1 j}+I_{2 j}+I_{3 j}+I_{4 j}+I_{5 j}\right) \text { for } 0<\varepsilon \leqslant \varepsilon_{2},
$$

in which the integrals $I_{1 j}, I_{2 j}, I_{3 j}, I_{4 j}$ and $I_{5 j}$ of $1 / \Phi_{k}$ are taken on the intervals

$$
\begin{array}{cc}
{\left[-2 j \pi-\pi / 2+\sigma,-2 j \pi+\theta_{k}^{\circ}\right],} & {[-2 j \pi-\pi / 2-\sigma,-2 j \pi-\pi / 2+\sigma],} \\
{[-2 j \pi-3 \pi / 2+\sigma,-2 j \pi-\pi / 2-\sigma],} & {[-2 j \pi-3 \pi / 2-\sigma,-2 j \pi-3 \pi / 2+\sigma]}
\end{array}
$$

and

$$
\left[-2(j+1) \pi+\theta_{k}^{\circ},-2 j \pi-3 \pi / 2-\sigma\right],
$$

respectively, and $\sigma=A \pi \varepsilon$ and $j=0,1, \ldots, m_{k}-1$. Here we assume $\theta_{k}^{\circ} \in[-\pi / 2+$ $\sigma, \pi / 2-\sigma]$ for the definiteness, and the proof is also applicable to the other cases.

For simplicity, we set

$$
\omega_{k}=\int_{\theta_{k}^{\circ}-2 m_{k} \pi}^{\theta_{k}^{\circ}} \frac{p_{k}(t)}{\gamma_{k}} \cos \theta_{k} d \theta_{k}=\sum_{j=0}^{m_{k}-1}\left(H_{1 j}+H_{2 j}+H_{3 j}+H_{4 j}+H_{5 j}\right)
$$

in which $H_{i j}$ has the same interval of integration as that of $I_{i j}(i=1,2,3,4,5 ; j=$ $\left.0,1, \ldots, m_{k}-1\right)$, respectively.

For $1-\delta \leqslant \gamma_{k} \leqslant 1+\delta$ and

$$
-2 j \pi-\pi / 2+\sigma \leqslant \theta_{k} \leqslant-2 j \pi+\theta_{k}^{\circ} \leqslant-2 j \pi+\pi / 2-\sigma,
$$

we have

$$
\frac{u_{k}}{\varepsilon}=\frac{\gamma_{k} \cos \theta_{k}}{\varepsilon} \geqslant \frac{1-\delta}{\varepsilon} \sin \sigma \geqslant \frac{2(1-\delta) \sigma}{\pi \varepsilon}>\frac{\sigma}{\pi \varepsilon}=A
$$


Hence, by $\left(\mathrm{H}_{2}\right)$, it is not hard to derive the following inequalities:

$$
\begin{aligned}
I_{1 j}>\frac{1}{m_{k}} \int_{-2 j \pi-\pi / 2+\sigma}^{-2 j \pi+\theta_{k}^{\circ}}\left[1-B_{k}\left(\frac{\varepsilon}{m_{k} \gamma_{k}}\right)^{2} \frac{\gamma_{k} \cos \theta_{k}}{\varepsilon} \tan ^{-1}\left(\frac{\gamma_{k} \cos \theta_{k}}{\varepsilon}\right)\right. \\
>\frac{1}{m_{k}} \int_{-\pi / 2+\sigma}^{\theta_{k}^{\circ}}\left[1+\frac{\varepsilon(1-\delta) B_{k}}{m_{k}^{2} \gamma_{k}} p_{k}(t) \cos \theta_{k}\right]^{-1} d \theta_{k} \\
\quad+\frac{\varepsilon}{m_{k}^{3}} H_{1 j}+O\left(\varepsilon^{2}\right),
\end{aligned}
$$

and

$$
\begin{aligned}
I_{5 j}> & \frac{1}{m_{k}} \int_{\theta_{k}^{\circ}}^{\pi / 2-\sigma}\left[1+\frac{\varepsilon(1-\delta) B_{k}}{(1+\delta)^{2} m_{k}^{2}} \cos \theta_{k} \cdot \tan ^{-1}\left(\frac{1-\delta}{\varepsilon} \cos \theta_{k}\right)\right] d \theta_{k} \\
& +\frac{\varepsilon}{m_{k}^{3}} H_{5 j}+O\left(\varepsilon^{2}\right) .
\end{aligned}
$$

Then we have

$$
\begin{aligned}
I_{1 j}+I_{5 j}> & \frac{1}{m_{k}} \int_{-\pi / 2+\sigma}^{\pi / 2-\sigma}\left[1+\frac{\varepsilon(1-\delta) B_{k}}{(1+\delta)^{2} m_{k}^{2}} \cos \theta_{k} \cdot \tan ^{-1}\left(\frac{1-\delta}{\varepsilon} \cos \theta_{k}\right)\right] d \theta_{k} \\
& +\frac{\varepsilon}{m_{k}^{3}}\left(H_{1 j}+H_{5 j}\right)+O\left(\varepsilon^{2}\right) \\
= & \frac{1}{m_{k}}\left[(\pi-2 \sigma)+\frac{\varepsilon(1-\delta) B_{k}}{(1+\delta)^{2} m_{k}^{2}} J(\varepsilon)\right]+\frac{\varepsilon}{m_{k}^{3}}\left(H_{1 j}+H_{5 j}\right)+O\left(\varepsilon^{2}\right) .
\end{aligned}
$$

Hence, it follows from Lemma 2 that

$$
I_{1 j}+I_{5 j}>\frac{1}{m_{k}}\left[(\pi-2 \sigma)+\frac{\varepsilon(1-\delta) B_{k} \pi}{(1+\delta)^{2} m_{k}^{2}}\right]+\frac{\varepsilon}{m_{k}^{3}}\left(H_{1 j}+H_{5 j}\right)+O\left(\varepsilon^{2}\right) .
$$

By using the same technique and the corollary of Lemma 2, we have

$$
I_{3 j}>\frac{1}{m_{k}}\left[(\pi-2 \sigma)+\frac{\varepsilon(1-\delta) B_{k} \pi}{(1+\delta)^{2} m_{k}^{2}}\right]+\frac{\varepsilon}{m_{k}^{3}} H_{3 j}+O\left(\varepsilon^{2}\right) .
$$

On the other hand, since $H_{2 j}=O(\varepsilon)$ and $H_{4 j}=O(\varepsilon)$, we can write

$$
I_{2 j}=2 \sigma / m_{k}+\left(\varepsilon / m_{k}^{3}\right) H_{2 j}+O\left(\varepsilon^{2}\right),
$$

and

$$
I_{4 j}=2 \sigma / m_{k}+\left(\varepsilon / m_{k}^{3}\right) H_{4 j}+O\left(\varepsilon^{2}\right) .
$$


We thus obtain the inequality

$$
\begin{aligned}
\tau_{k} & >\frac{1}{m_{k}} \sum_{j=0}^{m_{k}-1}\left[2(\pi-2 \sigma)+\frac{2 \varepsilon(1-\delta) B_{k} \pi}{(1+\delta)^{2} m_{k}^{2}}+4 \sigma\right]+\frac{\varepsilon}{m_{k}^{3}} \omega_{k}+O\left(\varepsilon^{2}\right) \\
& =2 \pi+\frac{2 \varepsilon(1-\delta) B_{k} \pi}{(1+\delta)^{2} m_{k}^{2}}+\frac{\varepsilon}{m_{k}^{3}} \omega_{k}+O\left(\varepsilon^{2}\right) .
\end{aligned}
$$

Since

$$
\omega_{k}=\int_{\theta_{k}^{\circ}-2 m_{k} \pi}^{\theta_{k}^{\circ}} \frac{p_{k}(t)}{\gamma_{k}} \cos \theta_{k} d \theta_{k}=\int_{0}^{\tau_{k}} \frac{p_{k}(t) \cos \theta_{k}}{\gamma_{k}} \Phi_{k} d t
$$

and

$$
\lim _{\varepsilon \rightarrow 0}\left(\tau_{k}, \theta_{k}, \gamma_{k}, \Phi_{k}\right)=\left(2 \pi,-m_{k} t+\theta_{k}^{\circ}, 1, m_{k}\right)
$$

we get

$$
\begin{aligned}
\omega_{k} & =\int_{0}^{2 \pi} m_{k} p_{k}(t) \cos \left(-m_{k} t+\theta_{k}^{\circ}\right) d t+\zeta(\varepsilon) \\
& \geqslant-2 m_{k} \pi\left(E_{k}^{\circ}+F_{k}^{\circ}\right)+\zeta(\varepsilon),
\end{aligned}
$$

where $\zeta(\varepsilon)$ is continuous in $\varepsilon(0 \leqslant \varepsilon \ll 1)$ and $\zeta(0)=0$. Hence, we arrive at the inequality

$$
\tau_{k}>2 \pi+\frac{2 \pi \varepsilon}{m_{k}^{2}}\left[\frac{(1-\delta) B_{k}}{(1+\delta)^{2}}-\left(E_{k}^{\circ}+F_{k}^{\circ}\right)\right]+\frac{\varepsilon \zeta(\varepsilon)}{m_{k}^{3}}+O\left(\varepsilon^{2}\right) .
$$

The inequalities (4.1) and (4.2) guarantee the existence of a positive constant $\varepsilon_{3}$ such that for any $\varepsilon\left(0<\varepsilon \leqslant \varepsilon_{3}\right)$ and $(\xi, \eta) \in \Gamma_{k}$, the inequality $\tau_{k}>2 \pi$ is valid. It means that the time interval $\left[0, \tau_{k}\right]$, in which the projection $\left(u_{k}, v_{k}\right)$ of the motion (3.5) will complete $m_{k}$ rotations around the origin $O_{k}$, is longer than $2 \pi$. Hence, in the shorter interval $[0,2 \pi]$, the projection $\left(u_{k}, v_{k}\right)$ can merely finish some rotations less than $m_{k}$, i.e.,

$$
\Delta \theta_{k}=\theta_{k}(2 \pi, \xi, \eta, \varepsilon)-\theta_{k}^{\circ}>-2 m_{k} \pi,
$$

and the proof of Lemma 5 is thus completed.

Finally, let us consider a variant form of $\left(\mathrm{H}_{2}\right)$.

$\left(\mathrm{H}_{2}^{*}\right)$ There exist positive constants $A$ and $B_{j}(j=1,2, \ldots, n)$ such that when $t \in[0,2 \pi]$ and $\left|x_{j}\right| \geqslant A$, the inequalities

$$
x_{j} f_{j}(t, x) \geqslant B_{j} x_{j} \tan ^{-1} x_{j} \quad(j=1,2, \ldots, n)
$$

hold.

Then, in a similar way, we can prove the following lemma:

LEMMA $5^{*}$. If $\left(\mathrm{H}_{1}\right),\left(\mathrm{H}_{2}^{*}\right)$ and $\left(\mathrm{H}_{3}\right)$ hold, then there is a positive constant $\varepsilon_{3}^{*}$ such that for any $\varepsilon\left(0<\varepsilon \leqslant \varepsilon_{3}^{*}\right)$ and $(\xi, \eta) \in \Gamma_{k}$, the increment

$$
\Delta \theta_{k}=\theta_{k}(2 \pi, \xi, \eta, \varepsilon)-\theta_{k}^{\circ}<-2 m_{k} \pi \quad(k=1,2, \ldots, n) .
$$


5. We are now in a position to prove our main theorems.

THEOREM 1. If $\left(\mathrm{H}_{1}\right),\left(\mathrm{H}_{2}\right)$ and $\left(\mathrm{H}_{3}\right)$ hold, then the differential system (1.4) has at least one $2 \pi$-periodic solution.

Proof. Consider the mapping

$$
T_{\varepsilon}: G \rightarrow R^{2 n} \quad(0<\varepsilon \ll 1),
$$

defined in $\S 3$.

It follows from Lemmas 4 and 5 that

$$
\begin{aligned}
\left(\bar{u}_{k}-\xi_{k}\right) \xi_{k} & +\left(\bar{v}_{k}-\eta_{k}\right) \eta_{k} \\
& \neq \sqrt{\xi_{k}^{2}+\eta_{k}^{2}} \cdot \sqrt{\left(\bar{u}_{k}-\xi_{k}\right)^{2}+\left(\bar{v}_{k}-\eta_{k}\right)^{2}}, \quad(\xi, \eta) \in \Gamma_{k}
\end{aligned}
$$

$(k=1,2, \ldots, n)$. Hence, by Lemma 1 , we can conclude that $T_{\varepsilon}$ has at least one fixed point, say $\left(\xi^{*}, \eta^{*}\right)$, in $G$. Hence,

$$
u=u\left(t, \xi^{*}, \eta^{*}, \varepsilon\right), \quad v=v\left(t, \xi^{*}, \eta^{*}, \varepsilon\right)
$$

is a $2 \pi$-periodic solution of $(3.3)$, and then

$$
x=\varepsilon^{-1} u\left(t, \xi^{*}, \eta^{*}, \varepsilon\right)
$$

is a $2 \pi$-periodic solution of (1.4). The proof of Theorem 1 is thus completed.

By using Lemmas 4 and $5^{*}$, we can prove the following theorem:

THEOREM 2. If $\left(\mathrm{H}_{1}\right),\left(\mathrm{H}_{2}^{*}\right)$ and $\left(\mathrm{H}_{3}\right)$ hold, then the differential system (1.4) has at least one $2 \pi$-periodic solution.

I am grateful to Professor George R. Sell for his many helpful suggestions.

\section{REFERENCES}

1. A. C. Lazer, On Schauder's fixed point theorem and forced second-order nonlinear oscillations, J. Math. Anal. Appl. 21 (1968), 421-425.

2. J. Mawhin, An extension of a theorem of A.C. Lazer on forced nonlinear oscillations, J. Math. Anal. Appl. 40 (1972), 20-29.

3. A. C. Lazer and D. E. Leach, Bounded perturbations of forced harmonic oscillations at resonance, Ann. Mat. Pura Appl. (4) 82 (1969), 49-68.

4. L. Césari, Nonlinear problems across a point of resonance for non-self-adjoint sistems, Nonlinear Analysis (A Collection of Papers in Honor of Erich H. Rothe, edited by L.Césari, et al.), Academic Press, New York, 1978.

5. Tung-ren Ding, Nonlinear oscillations at a point of resonance, Sci. Sinica (Ser. A) 25 (1982), 918-931.

6. Some fixed point theorems and periodically perturbed non-dissipative system. Ann. of Math. (2) 2 (1981), 281-297.

Department of Mathematics, Peking University, Beijing, China 